\title{
NUMERICAL DETERMINATION OF THE PARITY-CONDITION PARAMETER FOR LANCHESTER-TYPE EQUATIONS OF MODERN WARFARE*
}

\author{
James G. TAYLOR $\dagger$ and Gerald G. Brown $\ddagger$ \\ Departments of Operations Research and Computer Science, Naval Postgraduate School, Monterey, \\ California
}

\begin{abstract}
Scope and purpose-This paper presents new computational methods that facilitate digital-computer analysis of some important military operations research problems. Lanchester-type combat models[1] are deterministic differential-equation models of combat attrition in which the state variables are typically the numbers of the different weapon-system types. Even though combat between two military forces is a complex random process, such deterministic combat models are commonly used for computational reasons in defense-planning studies, for example, to assess the relative importance of various weapon-system and force-level parameters. A so-called attrition-rate coefficient in such a combat model represents the fire effectiveness of a particular weapon-system type against a particular target type, and time-dependent attrition-rate coefficients are used to model temporal variations in fire effectiveness when, for example, the range between firers and targets changes appreciably during battle. For such a variable-coefficient Lanchester-type combat model that is a generalization of Lanchester's [2] classic "square-law" model, we present a simple numerical procedure for determining the so-called parity-condition parameter, which is "the enemy force equivalent of a friendly force of unit strength" and may be used to predict battle outcome in specific engagements. These results allow one not only to predict battle outcome but also to tradeoff quality vs quantity of two opposing weapon systems.
\end{abstract}

Abstract-This paper presents a simple numerical procedure for determining the parity-condition parameter for deterministic Lanchester-type combat between two homogeneous forces. Deterministic differentialequation combat models are commonly used in parametric studies for computational reasons, since they give essentially the same results for the mean course of combat as do corresponding stochastic attrition models. The combat studied in this paper is modelled by Lanchester-type equations of modern warfare with time-dependent attrition-rate coefficients. Previous research has generalized Lanchester's classic "square law" to such variable-coefficient combat. It has shown that the prediction of battle outcome (in particular, force annihilation) without having to spend the time and effort of computing force-level trajectories depends on a single parameter, the so-called parity-condition parameter, which is "the enemy force equivalent of a friendly force of unit strength" and depends on only the attrition-rate coefficients. Unfortunately, previous research did not show generally how to determine this parameter. We present general theoretical considerations for its numerical noniterative determination. This general theory is applied to an important class of attrition-rate coefficients (offset power-rate coefficients). Our results allow one to study such variable-coefficient combat models almost as easily and thoroughly as Lanchester's classic constant-coefficient model.

\section{INTRODUCTION}

As a consequence of pioneering work by F. W. Lanchester done about the time of World War I, military analysts have used simplified deterministic differential-equation models to develop insights into the dynamics of combat from about the end of World War II (see, for example, [1, 3-11]. Such deterministic models have been widely used because (among other reasons) the

*This research was partially supported by the Office of Naval Research and by the U.S. Army Research Office, Durham, North Carolina, under R\&D Project No. 1L161102H57-05 Math.

†James G. Taylor is an Associate Professor of Operations Research at the U.S. Naval Postgraduate School (NPS). He received B.S., M.S., and Ph.D. degrees in Petroleum Engineering from Stanford University. He is the author of over twenty publications on various aspects of operations research and was awarded (jointly with S. Parry) the 1975 MAS Prize by the Military Applications Section (MAS) of the Operations Research Society of America (ORSA). He is the co-author with Professor Gerald G. Brown of several papers on Lanchester-type models and optimal control theory. Professor Taylor's recent research has been on the Lanchester theory of combat and generalized control theory. He serves as an Associate Editor of both Operations Research and Naval Research Logistics Quarterly and also as a Councilman of the Military Applications Section of ORSA.

$\ddagger$ Gerald G. Brown is an Associate Professor of Operations Research and Computer Science at the Naval Postgraduate School, Monterey. He earned his Ph.D. from the University of California, Los Angeles, and is a member of ACM, AIIE, ASA, MORS, ORSA and TIMS. He has published articles in the journals and proceedings of those societies and in Mathematics Teacher, IEEE Transactions and Naval Research Logistics Quarterly. His current research concentrates on heavily computational management science models, with emphasis on large scale optimization. 
corresponding stochastic formulations are for all practical purposes analytically intractable (see Note 1 on p. 65 of [12]). The advent of the modern high-speed digital computer has made feasible the development and use of quite complicated versions of such Lanchester-type* models as practical defense planning tools [13]. Thus, today militarily realistic computer-based Lanchestertype models of quite complex combat systems have been developed. Such models currently exist for almost the entire spectrum of combat operations, from combat between battalion-sized [14] and division-sized [15] units to theater-level operations $[16,17]$.

A simple combat model, however, may yield a clearer understanding of important interrelationships that are difficult to perceive in a more complex model, and such insights can provide valuable guidance for more detailed computerized investigations-(see [4, 11]). In this paper we present a new important numerical procedure that facilitates parametric analysis (in particular, the parametric examination of force-annihilation prediction) of battle outcomes for such simplified Lanchester-type models of combat between two homogeneous forces with temporal variations in each side's fire effectiveness. Previously, such battle-outcome information could only be readily obtained from constant-coefficient models, and $\mathrm{S}$. Bonder $[3,18,19]$ has emphasized the deficiencies of constant-coefficient models (see Section 3 below). These results are not only significant in their own right but are also useful in the quantitative analysis of time-sequential combat strategies (see, for example, [20,21]).

It is important for the military operations analyst to have a clear understanding of how force-level and weapon-system-performance parameters interact to determine a battle's outcome. Such knowledge is particularly useful in weapon-system and force-level planning activities for defense planning (especially since one frequently uses models that are so complicated that trends are not directly discernible without extensive (and costly) computer runs). S. Bonder's $[3,4,19]$ pioneering work on methodology for the evaluation of military systems (particularly mobile systems such as tanks, mechanized infantry combat vehicles, etc.) provides a motivation for interest in variable-coefficient, deterministic, Lanchester-type combat models such as we consider in this paper. He has stressed (see pp. 30-31 of [4]) the importance of analytical solutions to such models for developing insights into the dynamics of combat by portraying the relation between various factors in the combat attrition process and the surviving numbers of forces and for facilitating sensitivity and other parametric analyses (see [22]). Unfortunately, as work by Bonder and Farrell[4] and Taylor [12,23] shows, the analytical (i.e. infinite series) solution to variable-coefficient equations generally by itself (i.e. without explicitly computing force-level trajectories) provides little information about battle outcome because of its complexity. Therefore, one must seek new ways for developing insights.

Taylor and Comstock[7] have given results that allow one to predict battle outcome (in particular, force annihilation $\dagger$ ) in theory without having to spend the time and effort of computing force-level trajectories. To be computationally practical, however, their results require the determination of the so-called parity-condition parameter ("the enemy force equivalent of a friendly force of unit strength"), which depends on only the model's attritionrate coefficients. They analytically determine the parity-condition parameter for power attritionrate coefficients with "no offset", which allow one to model combat between two weapon systems with the same minimum effective range but different range dependencies for each system's fire effectiveness (see also [6]). It is the purpose of this paper to show how to determine the parity-condition parameter in other cases, in particular for power attrition-rate coefficients with "positive offset", which allow one to model such combat between weapon systems with different maximum effective ranges. Our results allow one to study in general such variable-coefficient combat models almost as easily and thoroughly as Lanchester's classic constant-coefficient model.

The organization of this paper is as follows. We first review Lanchester-type equations of modern warfare, especially variable-coefficient formulations. Next we review force-annihilation-prediction conditions for such models and show how to use knowledge about the

\footnotetext{
*Also frequently called differential models of combat[15].

$\dagger$ Bonder and Honig[5] point out, however, that force annihilation may not always be the best criterion for evaluating military operations. See pp. 192-242 of Bonder and Farrell [4] for a detailed Lanchester-type analysis of an attack scenario for which other "end of battle conditions" play the principal role. Nevertheless, it is of considerable interest (especially for developing insights into the dynamics of combat) to be able to easily predict the occurrence of force annihilation.
} 
parity-condition parameter for one set of attrition-rate coefficients to numerically determine it in related cases of interest. This general theory is then applied to the important case of offset power attrition-rate coefficients, with detailed numerical examples being given.

\section{LANCHESTER'S CLASSIC COMBAT FORMULATION}

F. W. Lanchester [2] (see also p. 45 of [12]) hypothesized in 1914 that combat between two military forces could be modelled by

$$
\mathrm{d} x / \mathrm{d} t=-a y, \quad \mathrm{~d} y / \mathrm{d} t=-b x,
$$

with initial conditions

$$
x(t=0)=x_{0}, \quad y(t=0)=y_{0},
$$

where $t=0$ denotes the time at which the battle begins, $x(t)$ and $y(t)$ denote the numbers of $X$ and $Y$ at time $t$, and (for a particular battle) $a$ and $b$ are nonnegative constants which are today called Lanchester attrition-rate coefficients and represent each side's fire effectiveness. The equations (1) are only valid for $x, y>0$. For example, the first becomes $\mathrm{d} x / \mathrm{d} t=0$ when $x=0$.

Because of Lanchester's pioneering work[2], we will refer to any differential-equation model of combat attrition as a Lanchester-type combat model or as a system of Lanchestertype differential equations (or sometimes simply as Lanchester-type equations). In particular, we will refer to (1) as (constant-coefficient) Lanchester-type equations of modern warfare. Other forms of Lanchester-type equations appear in the literature [1,23], but we will not consider these here. Various sets of physical circumstances have been hypothesized to yield them: for example: (a) both sides use aimed fire and target acquisition times are constant[10]; or (b) both sides use area fire and a constant density defense (see p. 345 of [1]).

From (1) Lanchester deduced his famous square law

$$
b\left(x_{0}^{2}-x^{2}(t)\right)=a\left(y_{0}^{2}-y^{2}(t)\right) .
$$

Consider now a battle terminated* by either force level reaching a given "breakpoint": for example, $Y$ wins when $x_{f}=x\left(t_{f}\right)=x_{B P}=f_{X}{ }^{B P} x_{0}$ but $y_{f}>y_{B P}=f_{Y}{ }^{B P} y_{0}$, where $t_{f}, x_{f}, y_{f}$ denote final values and $x_{B P}$ denotes $X$ 's breakpoint which is a given fraction $f_{X}{ }^{B P}$ of his initial strength. It follows from (3) that

$$
\dot{Y} \text { will win if and only if } \frac{x_{0}}{y_{0}}<\sqrt{\left(\frac{a\left\{1-\left(f_{Y}^{B P}\right)^{2}\right\}}{b\left\{1-\left(f_{X}^{B P}\right)^{2}\right\}}\right)}
$$

which for a fight-to-the-finish (i.e. $f_{X}{ }^{B P}=f_{Y}{ }^{B P}=0$ ) becomes the classic result

$$
Y \text { will win a fight-to-the-finish if an only if } \frac{x_{0}}{y_{0}}<\sqrt{ }\left(\frac{a}{b}\right) \text {. }
$$

Unfortunately, no relationship similar to (3) holds in general for variable attrition-rate coefficients except when $a(t) / b(t)=$ constant (see p. 48 of [12]). This paper, nevertheless, shows how (5) generalizes in these cases, but so far we have not been able to generalize (4). Recalling that the time history of the $X$ force level is given by

$$
x(t)=x_{0} \cosh \sqrt{ }(a b) t-y_{0} \sqrt{ }\left(\frac{a}{b}\right) \sinh \sqrt{ }(a b) t,
$$

we see that the battle trajectories depend on the two weapon-system-performance parameters: (I) the intensity of combat $\sqrt{ } a b$, and (II) the relative fire effectiveness $a / b$. Only the relative fire effectiveness, however, determines the battle's outcome [see (4) and (5) above]. [8]).

*The modelling of battle termination is a problem area in contemporary defense planning studies (see pp. 524-525 of 


\section{VARIABLE ATTRITION-RATE COEFFICIENTS}

Bonder [18] has pointed out that in many cases (for example, in the case of mobile weapon systems) the validity of the assumption of constant attrition-rate coefficients is open to question (see also $[3,4,19])$. Thus, we consider

$$
\mathrm{d} x / \mathrm{d} t=-a(t) y, \quad \mathrm{~d} y / \mathrm{d} t=-b(t) x,
$$

where $a(t)$ and $b(t)$ denote time-dependent attrition-rate coefficients. We assume that $a(t)$ and $b(t)$ are defined, positive, and continuous for $t_{0}<t<+\infty$ with $t_{0} \leq 0$. For convenience, we introduce the notation that $a(t) \in L\left(t_{0}, T\right)$ means $\int_{t_{0}}^{T} a(t) \mathrm{d} t$. exists (and is given by a finite quantity). From our assumptions about $a(t)$ and $b(t)$, it follows that $a(t) \notin L\left(t_{0}, T\right)$ implies that $\int_{t_{0}}^{T} a(t) \mathrm{d} t=+\infty$. We also assume that $a(t), b(t) \in L\left(t_{0}, T\right)$ for any finite $T$. We further take $a(t)$ and $b(t)$ to be given in the form $a(t)=k_{a} g(t), b(t)=k_{b} h(t)$, where $k_{a}, k_{b}$ are positive constants chosen so that $a(t) / b(t)=k_{a} / k_{b}$ when $g(t)=h(t)$. Analogous to the constant-coefficient case [see discussion after (6)], we have the two weapon-system-performance parameters: (I) the intensity of combat, $I(t)=\sqrt{ }(a(t) b(t))$; and (II) the relative fire effectiveness, $R(t)=a(t) \mid b(t)$. We accordingly introduce the combat-intensity parameter $\lambda_{I}$ and the relative-fire-effectiveness parameter $\lambda_{R}$ defined by

$$
\lambda_{I}=\sqrt{ }\left(k_{a} k_{b}\right) \text { and } \lambda_{R}=k_{a} / k_{b} \text {. }
$$

Two significant developments in the Lanchester theory of combat during the 1960s were the development of methodology for (a) the prediction of Lanchester attrition-rate coefficients from weapon-system-performance data by S. Bonder $[18,24]$ and (b) the (maximum likelihood) estimation of such coefficients from Monte Carlo simulation output by G. Clark [25]. Both these developments and others (see [12] for further references) have generated interest in the model (7) and facilitated its application (and that of its generalization to combat between heterogeneous forces [4) to defense planning studies.

A large class of tactical situations of interest can be modelled with the following general power attrition-rate coefficients $[4,7,12]$

$$
a(t)=k_{a}(t+C)^{\mu}, \text { and } b(t)=k_{b}(t+C+A)^{\nu},
$$

where $A, C \geq 0$. We will call $A$ the offset parameter, since it allows us to model (with $\mu, \nu \geq 0$ ) battles between weapon systems with different maximum effective ranges. We will call $C$ the starting parameter, since it allows us to model (again with $\mu, \nu \geq 0$ ) battles that begin within the minimum of the maximum effective ranges of the two systems. For example, let us consider Bonder's [3, 19] model of a constant-speed attack on a static defensive position (see also $[12,23])$. Then we have

$$
\mathrm{d} x / \mathrm{d} t=-\alpha(r) y, \quad \mathrm{~d} y / \mathrm{d} t=-\beta(r) x,
$$

where $r(t)=R_{0}-v t$ denotes the distance (range) between the two opposing forces, $R_{0}$ denotes the battle's opening range, $v>0$ denotes the constant attack speed,

$$
\alpha(r)= \begin{cases}0 & \text { for } r \geq R_{\alpha}, \\ \alpha_{0}\left(1-r / R_{\alpha}\right)^{\mu} & \text { for } 0 \leq r \leq R_{\alpha},\end{cases}
$$

$\dot{\mu} \geq 0$, and $R_{\alpha}$ denotes the maximum effective range of $Y$ 's weapon system. Similarly for $\beta(r)$, with exponent $\nu \geq 0$. In (11) the parameter $\mu$ allows us to model the range dependence of $Y$ 's fire effectiveness (see Fig. 1). The offset and starting parameters are given by

$$
A=\left(R_{\beta}-R_{\alpha}\right) / v \text {, and } C=\left(R_{\alpha}-R_{0}\right) / v \text {, }
$$

and the assumption $A, C \geq 0$ implies that $R_{\beta} \geq R_{\alpha} \geq R_{0}$. From considering (12) and Fig. 2, the reader should have no trouble understanding our terminology for $A$ and $C$. 


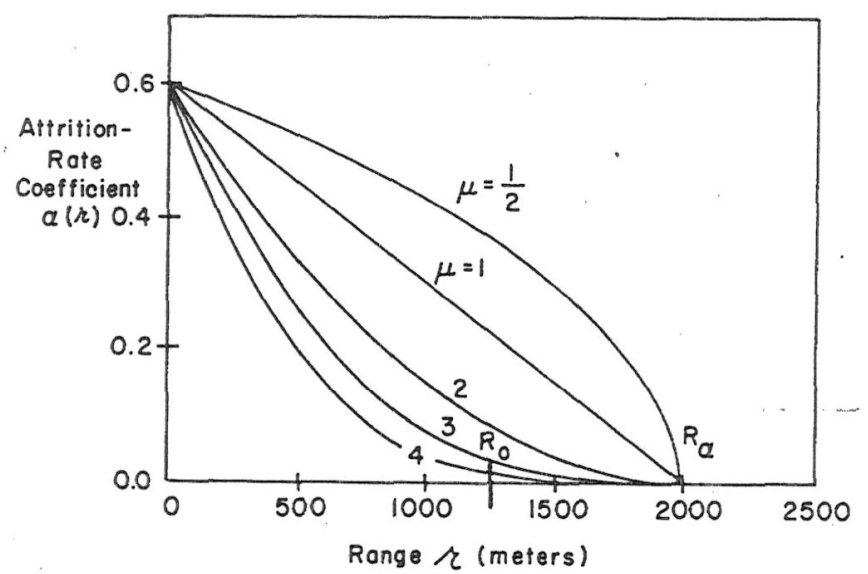

Fig. 1. Dependence of the attrition-rate coefficient $\alpha(r)$ on the exponent $\mu$ with maximum effective range of the weapon system and kill capability at zero range held constant. (Notes: (1) the maximum effective range of the system is denoted as $R_{\alpha}=2000 \mathrm{~m} ;(2) \alpha(r=0)=\alpha_{0}=0.6 X$ casualties/(unit time $\times$ number of $Y$ units) denotes the $Y$-force weapon-system kill rate at zero force separation (denoted here as range); (3) the opening range of battle is denoted as $R_{0}=1250 \mathrm{~m}$ and (as shown) $R_{0}<R_{\alpha}$.)

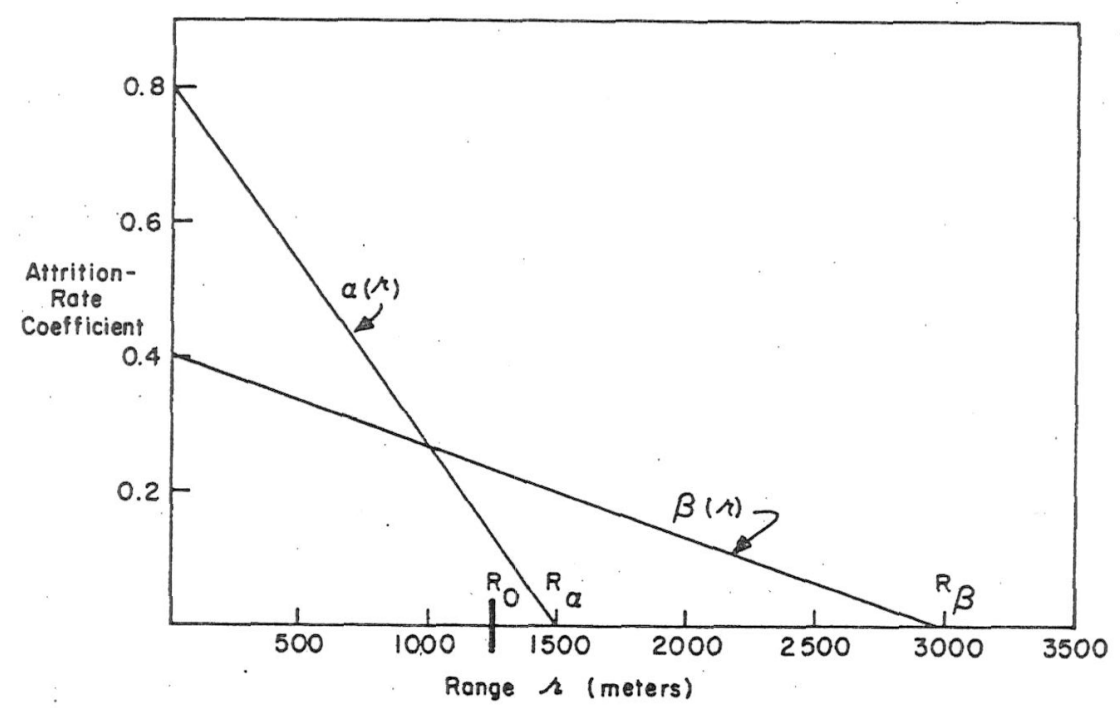

Fig. 2. Explanation of offset parameter $A$ and starting parameter $C$ for power attrition-rate coefficients modelling constant-speed attack. Notes: (1) the maximum effective ranges of the two weapon systems are denoted as $R_{\alpha}$ and $R_{\beta}$; (2) the opening range of battle (i.e. initial separation between forces) is denoted as $R_{0}$ and, as shown, $R_{0}<$ Minimum $\left(R_{\alpha}, R_{\beta}\right)$; (3) the offset parameter is given by $A=\left(R_{\beta}-R_{\alpha}^{\alpha} \% / v ;(4)\right.$ the starting parameter is given by $C=\left(R_{\alpha}-R_{0}\right) / v$.]

The time history of the $X$ force level, i.e. the solution $x(t)$ to (7), is given by [12]

$$
x(t)=x_{0}\left\{C_{Y}(0) C_{X}(t)-S_{Y}(0) S_{X}(t)\right\}-y_{0} V\left(\lambda_{R}\right)\left\{C_{X}(0) S_{X}(t)-S_{X}(0) C_{X}(t)\right\}
$$

where the hyperbolic-like general Lanchester functions (GLF) $C_{X}(t)$ and $S_{X}(t)$ are linearly independent solutions to the $X$ force-level equation

$$
\frac{\mathrm{d}^{2} x}{\mathrm{~d} t^{2}}-\left\{\frac{1}{a(t)} \frac{\mathrm{d} a}{\mathrm{~d} t}\right\} \frac{\mathrm{d} x}{\mathrm{~d} t}-a(t) b(t) x=0
$$

with initial conditions

$$
\begin{gathered}
C_{X}\left(t=t_{0}\right)=1, \quad\left\{t=t_{0}\right)=0, \\
\left\{[1 / a(t)] \mathrm{d} C_{X} / \mathrm{d} t(t)\right\}_{t=t_{0}}=0, \quad\left\{[1 / a(t)] \mathrm{d} S_{X} / \mathrm{d} t(t)\right\}_{t=t_{0}}=1 / \sqrt{ }\left(\lambda_{R}\right),
\end{gathered}
$$


where $t_{0}$ denotes the la.gest finite time at which $a(t)$ or $b(t)$ ceases to be defined, positive, or continuous. More precisely, $t_{0}=\sup \left\{t_{1} \mid\right.$ either $a(t)$ or $b(t)$ is not defined, positive, or continuous for some finite $\left.t \geq t_{1}\right\}=\inf \left\{t_{1} \mid\right.$ both $a(t)$ and $b(t)$ are defined, positive, and continuous for all finite $\left.t>t_{0}\right\}$. For example, $t_{0}=-C$ for the general power attrition-rate coefficients (9). The time history of the $Y$ force level may be similarly obtained, with $C_{Y}(t)$ and $S_{Y}(t)$ being analogously defined for the corresponding $Y$ force-level equation.

For the numerical determination of the parity-condition parameter, it is convenient to introduce a new independent variable $s$ defined by

$$
s=K \lambda_{I} \int_{t_{0}}^{t} g(\sigma) \mathrm{d} \sigma
$$

where the parameter $K$ is to be chosen to simplify the form of $J(s)$ given by (18). We denote $s(t=0)$ as $s_{0}$, and then $s_{0} \geq 0$ if and only if $t_{0} \leq 0$. The substitution (16) transforms (14) into the normal form

$$
\frac{\mathrm{d}^{2} x}{\mathrm{~d} s^{2}}-J(s) x=0
$$

where

$$
J(s)=\frac{1}{K^{2}}\left\{\frac{h(t)}{g(t)}\right\}
$$

and $t=t(s)$. We also define the normal-form hyperbolic-like $G L F c_{X}(s)$ and $s_{X}(s)$, which satisfy (17) and the initial conditions

$$
\begin{gathered}
c_{X}(s=0)=1, \quad s_{X}(s=0)=0, \\
\mathrm{~d} c_{X} / \mathrm{d} s(s=0)=0, \quad \mathrm{~d} s_{X} / \mathrm{d} s(s=0)=1 .
\end{gathered}
$$

It follows that

$$
c_{X}(s(t))=C_{X}(t), \text { and } \quad s_{X}(s(t))=K S_{X}(t)
$$

\section{FORCE-ANNIHILATION-PREDICTION CONDITIONS}

Recently, Taylor and Comstock [7] have generalized the constant-coefficient force-annihilation-prediction condition (5) to battles modelled with Lanchester-type equations of modern warfare with time-dependent attrition-rate coefficients (7). In some sense their results generalize Lanchester's famous square law to variable-coefficient combat. Taylor and Comstock have shown that for the model (7) force-annihilation prediction involves (besides the initial force ratio $x_{0} / y_{0}$ and a relative-fire-effectiveness parameter $\lambda_{R}$ ) a single parameter, which they call the parity-condition parameter, denoted here as $Q^{*}$. Their main theoretical result is stated here for the reader's convenience as Theorem 1.

\section{Theorem 1}

Taylor and Comstock [7], assume that either $a(t) \notin L(0,+\infty)$ or $b(t) \notin L(0,+\infty)$. Then the $X$ force will be annihilated in finite time if and only if

$$
\frac{x_{0}}{y_{0}}<\sqrt{ }\left(\lambda_{R}\right)\left\{\frac{C_{X}(0)-Q^{*} S_{X}(0)}{Q^{*} C_{Y}(0)-S_{Y}(0)}\right\}
$$

where the parity-condition parameter $Q^{*}$ is unique and given by

$$
\lim _{t \rightarrow+\infty} \frac{S_{X}(t)}{C_{X}(t)}=\frac{1}{Q^{*}} .
$$


Remark 1. The condition (21) means that neither force is annihilated (in other words, the forces are of "equal fighting strength") if and only if .

$$
\frac{x_{0}}{y_{0}}=\sqrt{ }\left(\lambda_{R}\right)\left\{\frac{C_{X}(0)-Q^{*} S_{X}(0)}{Q^{*} C_{Y}(0)-S_{Y}(0)}\right\}
$$

which when $t_{0}=0$, simplifies to

$$
\frac{x_{0}}{y_{0}}=\frac{\sqrt[V]{ }\left(\lambda_{R}\right)}{Q^{*}}
$$

In other words, the above equation (23) is a condition under which two forces are "at parity" with one another (hence, the term parity-condition parameter). Observing the special case (24), we may consider the parity-condition parameter to be "the enemy force equivalent of a friendly of unit strength" (see also equation (17) of [7]).

Remark 2. We also have $\lim _{t \rightarrow+\infty}\left\{S_{Y}(t) / C_{Y}(t)\right\}=Q^{*}$.

Remark 3. When $t_{0}=0,(21)$ simplifies: $X$ will be annihilated in finite time if and only if $x_{0} / y_{0}<\sqrt{ }\left(\lambda_{R}\right) / Q^{*}$.

Remark 4. The result (22) suggests a numerical procedure for approximately determining the parity-condition parameter $Q^{*}$ : we may approximate the parity-condition parameter $Q^{*}$ by $\hat{Q}=1 /\left\{S_{X}(\hat{t}) / C_{X}(\hat{t})\right\}$, where $\hat{t}$ is a "suitably large" value of $t$. In other words, we may estimate $Q^{*}$ simply by picking a large value for $t$ (we denote this selected large value by $\hat{t}$ ), computing $S_{X}(\hat{t})$ and $C_{X}(\hat{t})$, and then forming their ratio. Our estimate for $Q^{*}$ is then given by $\hat{Q}=$ $1 /\left\{S_{X}(\hat{t}) / C_{X}(\hat{t})\right\}$. The only problem is that we do not know how large to take $\hat{t}$ for "satisfactory" estimation of $Q^{*}$ : There is an estimation error, $E(\hat{t})=Q^{*}-\hat{Q}(\hat{t})$, which depends monotonically on $\hat{t}$, and a priori we do not know how large this error is. The present paper develops a bound on the magnitude of this error, and our new error estimate allows the goodness of approximation to be easily evaluated in many cases of interest.

We may also determine the parity-condition parameter with the normal-form hyperbolic-like $\mathrm{GLF}$, since $\lim _{s \rightarrow+\infty}\left\{s_{X}(s) / c_{X}(s)\right\}=1 / Z^{*}=K / Q^{*}$, where $Z^{*}$ is called the modified paritycondition parameter. In fact, we will find it more convenient to do so. With this in mind, let us introduce the $Y$-functions $c_{Y}(s)$ and $s_{Y}(s)$ [corresponding to $c_{X}(s)$ and $s_{X}(s)$ ] defined by

$$
\mathrm{d} c_{Y} / \mathrm{d} s=J(s) s_{X}, \quad \mathrm{~d} s_{Y} / \mathrm{d} s=J(s) c_{X}
$$

with initial conditions

$$
c_{Y}(s=0)=1, \quad s_{Y}(s=0)=0
$$

It follows that $c_{Y}(s)$ and $s_{Y}(s)$ are linearly independent solutions to the modified $Y$ equation

$$
\frac{\mathrm{d}}{\mathrm{d} s}\left\{\frac{1}{J(s)} \frac{\mathrm{d} y}{\mathrm{~d} s}\right\}-y=0
$$

and

$$
c_{Y}(s(t))=C_{Y}(t), \quad s_{Y}(s(t))=(1 / K) S_{Y}(t) .
$$

In terms of the new time variable $s$ defined by (16), Theorem 1 reads as follows:

\section{Theorem 2}

Assume that either $a(t) \notin L(0,+\infty)$ or $b(t) \notin L(0,+\infty)$. Then the $X$ force will be annihilated in finite time if and only if

$$
\frac{x_{0}}{y_{0}}<\frac{V\left(\lambda_{R}\right)}{K}\left\{\frac{c_{X}\left(s_{0}\right)-Z^{*} s_{X}\left(s_{0}\right)}{Z^{*} c_{Y}\left(s_{0}\right)-s_{Y}\left(s_{0}\right)}\right\}
$$


where the modified time variable sis given by $(16)$, and $c_{X}(s), s_{X}(s), c_{Y}(s)$, and $s_{Y}(s)$ denote the normal-form hyperbolic-like GLF. The modified parity-condition parameter $Z^{*}$ is unique and given by

$$
\lim _{s \rightarrow+\infty} \frac{s_{X}(s)}{c_{X}(s)}=\frac{1}{Z^{*}}
$$

We observe that

$$
Q^{*}=K Z^{*},
$$

and $\lim _{s \rightarrow+\infty}\left\{s_{Y}(s) / c_{Y}(s)\right\}=Z^{*}$. When (27) holds, the time to annihilate $X$, denoted as $t_{a}{ }^{X}$, is determined by $x\left(t_{a}{ }^{X}\right)=0$. If we denote the quotient of the two normal-form hyperbolic-like GLF $s_{X}(x)$ and $c_{X}(s)$ as $\eta_{X}(s)$, then it follows from (13) that

$$
\eta_{X}\left(s\left(t_{a}^{X}\right)\right)=\frac{\left\{x_{0} c_{Y}\left(s_{0}\right)+y_{0}\left(\sqrt{ }\left(\lambda_{R}\right) / K\right) s_{X}\left(s_{0}\right)\right\}}{\left\{x_{0} s_{Y}\left(s_{0}\right)+y_{0}\left(\sqrt{ }\left(\lambda_{R}\right) / K\right) c_{X}\left(s_{0}\right)\right\}}
$$

where

$$
\eta_{X}(s)=s_{X}(s) / c_{X}(s)
$$

\section{DETERMINATION OF THE PARITY-CONDITION PARAMETER}

We will now show how knowledge about the modified parity-condition parameter $Z^{*}$ for one pair of attrition-rate coefficients, $a(t)$ and $b_{1}(t)$, allows us to determine $Z^{*}$ for a related pair, $a(t)$ and $b(t)$. With this in mind, let us denote $c_{X}(s)$ corresponding to $a(t)$ and $b(t)$ as $c_{X}(s ; a, b)$, and similarly for $s_{x}$ and $\eta_{x}$. In other words, we will now write (31) corresponding to the attrition-rate coefficients $a(t)$ and $b(t)$ as

$$
\eta_{X}(s ; a, b)=s_{X}(s ; a, b) / c_{X}(s ; a, b) .
$$

In this notation, we will write (28) as

$$
\lim _{s \rightarrow+\infty} \eta_{X}(s ; a, b)=1 / Z^{*}[a, b]
$$

We use the notation $Z^{*}[a, b]$ to show that the modified parity-condition parameter is a functional (i.e. a function for which the independent variables themselves are functions), which depends on only the attrition-rate coefficients $a(t)$ and $b(t)$. In other words, the attrition-rate coefficients are functions defined for $t_{0} \leq t<+\infty$, and the parity-condition parameter depends on these entire functions (and not merely particular values of them).

Our main result is Theorem 5, which gives an error estimate for the approximation that we propose for $Z^{*}$. The theoretical basis for Theorem 5 is given by Theorem 4, which (in turn) is a consequence of Theorem 3. The proof of Theorem 3 follows along the lines of well-known arguments (see p. 225 of [26]).

\section{Theorem 3}

Comparison Theorem: Let $x(t)$ and $x_{1}(t)$ satisfy

$$
\frac{\mathrm{d}}{\mathrm{d} t}\left\{\frac{1}{a(t)} \frac{\mathrm{d} x}{\mathrm{~d} t}\right\}-b(t) x=0, \quad \frac{\mathrm{d}}{\mathrm{d} t}\left\{\frac{1}{a(t)} \frac{\mathrm{d} x_{1}}{\mathrm{~d} t}\right\}-b_{1}(t) x_{1}=0,
$$

with initial conditions

$$
\begin{aligned}
& x\left(t=t_{0}\right)=\alpha, \quad x_{1}\left(t=t_{0}\right)=\alpha, \\
& \{[1 / a(t)] \mathrm{d} x / \mathrm{d} t(t)\}_{t=t_{0}}=\beta, \quad\left\{[1 / a(t)] \mathrm{d} x_{1} / \mathrm{d} t(t)\right\}_{t=t_{0}}=\beta,
\end{aligned}
$$

where $a(t)>0$ and $b_{1}(t)<b(t)$ for all $t>t_{0}$. Then $x_{1}(t)<x(t)$ for all $t>t_{0}$ as long as $x(t)>0$.

The basic theoretical result upon which our numerical determination of $Z^{*}$ is based is: 
Theorem 4.

Assume that $b_{1}(t)<b(t)$ for all $t>t_{0}$. Then

$$
\eta_{X}(s ; a, b)<1 / Z^{*}[a, b]<\eta_{X}(s ; a, b)+\left\{\left(1 / Z^{*}\left[a, b_{1}\right]\right)-\eta_{X}\left(s ; a, b_{1}\right)\right\} .
$$

Proof. We observe that $[7] \eta_{X}(s ; a, b)$ satisfies the differential equation

$$
\mathrm{d} \eta_{X} / \mathrm{d} s(s ; a, b)=1 /\left\{c_{X}(s ; a, b)\right\}^{2}
$$

with $\eta_{X}(s=0 ; a, b)=0$, and similarly for $\eta_{X}\left(s ; a, b_{1}\right)$. Theorem 3 (the comparison theorem) yields that $c_{X}(s ; a, b)>c_{X}\left(s ; a, b_{1}\right)$ for all $s>0$. Thus, for all $s>0$

$$
\mathrm{d} \eta_{X} / \mathrm{d} s(s ; a, b)<\mathrm{d} \eta_{X} / \mathrm{d} s\left(s ; a, b_{1}\right),
$$

whence integration between 0 and $s$ yields the desired result. Q.E.D.

Similar to the observations made in Remark 4 above, we observe that (33) suggests that we estimate $Z *[a, b]$ with $\hat{Z}$ defined by

$$
\hat{Z}(\hat{s} ; a, b)=1 / \eta_{X}(\hat{s} ; a, b),
$$

where $\hat{s}$ denotes a suitably chosen value for $s$. Moreover, from (35) we see that $\eta_{X}(s ; a, b)$ is a strictly increasing function of $s$ so that the larger we take $\hat{s}$ in (36), the better our approximation becomes. The only problem (see Remark 4 ) is that a priori we do not know how large to take $\hat{s}$ for "satisfactory" estimation of $Z^{*}$. Theorem 5 , however, tells us exactly how large to take $\hat{s}$.

\section{Theorem 5}

Error Estimate for Approximation: Assume that $b_{1}(t)<b(t)$ for all $t>t_{0}$. Let $f_{E}(\hat{s})$ denote the fractional error made in the estimation of $Z^{*}[a, b]$ by $\hat{Z}(\hat{s} ; a, b)$, i.e.

$$
f_{E}(\hat{s})=\frac{\hat{Z}(\hat{s} ; a, b)-Z^{*}[a, b]}{Z^{*}[a, b]}
$$

inen

$$
0<f_{E}(\hat{s})<\left\{\left(1 / Z *\left[a, b_{1}\right]\right)-\eta_{X}\left(\hat{s} ; a, b_{1}\right)\right\} \cdot \hat{Z}(\hat{s} ; a, b) .
$$

Proof. The theorem follows by simple algebraic manipulation after setting $s=\hat{s}$ in (34) and using (37). Q.E.D.

Thus, we have presented a method for numerically determining $Z *[a, b]$. We simply pick a large value for $s$ (we denote the selected value as $\hat{s}$ ), compute $s_{X}(\hat{s})$ and $c_{X}(\hat{s})$, and then compute the estimate $\hat{Z}(\hat{s} ; a, b)$ according to (36). Theorem 5 allows us to know the accuracy of our approximation, which can be improved by taking $\hat{s}$ larger. Thus, we can numerically determine $Z^{*}[a, b]$ to any specified degree of accuracy once $Z^{*}\left[a, b_{1}\right]$ is known. In the next section we apply this theory to the analysis of battles modelled with offset power attrition-rate coefficients.

\section{APPLICATION OF THEORY TO OFFSET POWER ATTRITION-RATE COEFFICIENTS}

In the application of Theorems 4 and 5, two pairs of attrition-rate coefficients are involved: one pair for which the modified parity-condition parameter is known [denoted as $a(t)$ and $b_{1}(t)$ ], and one for which it is to be determined [denoted as $a(t)$ and $b(t)$ ]. Accordingly, we rewrite (9) with $A>0$ as

$$
a(t)=k_{a}(t+C)^{\mu} \text {, and } b(t)=k_{b}(t+C+A)^{\nu},
$$

where (as before) $C \geq 0$. We will refer to these coefficients (39) for which $A>0$ as power attrition-rate coefficients with "positive offset". If we choose

$$
K=\left[\lambda_{I} /(\mu+1)\right]^{2 p-1},
$$


it follows from (16) that the modified time variable $s$ is given by

$$
s(t)=\left[\lambda_{I} /(\mu+1)\right]^{2 p}(t+C)^{\mu+1},
$$

and the invariant $J(s)$ of the normal form (17) simplifies to

$$
J(s ; a, b)=J(s ; \gamma, \mu, \nu)=s^{\beta}\left(1+\frac{\gamma}{s^{\alpha}}\right)
$$

where $p=(\mu+1) / \Sigma, \alpha=1 /(\mu+1), \beta=(\nu-\mu) /(\mu+1), \gamma=A \cdot\left[\lambda_{I} /(\mu+1)\right]^{2 / \Sigma}$, and $\Sigma=\mu+\nu+2$. Here we have denoted the invariant corresponding to the attrition-rate coefficients $a(t)$ and $b(t)$ as $J(s ; \gamma, \mu, \nu)$, since we may take $\gamma, \mu$, and $\nu$ as a basis for generating the four parameters $\alpha, \beta$, $\gamma$ and $\nu$ that explicitly appear in the right-hand side of (42). Furthermore, we will denote the normal-form hyperbolic-like GLF that correspond to $J(s ; \gamma, \mu, \nu)$ as $c_{X}(s ; \gamma, \mu, \nu)$ and $s_{X}(s ; \gamma, \mu, \nu)$.

The known results [7] that we use in the Theorems 4 and 5 are for the case of power attrition-rate coefficients with no offset [i.e. set $A=0$ in (9)]

$$
a(t)=k_{a}(t+C)^{\mu} \text {, and } b_{1}(t)=k_{b}(t+C)^{\nu},
$$

where $C \geq 0$. We observe that $b_{1}(t)<b(t)$ for all $t>-C$. It follows that $J\left(s ; a, b_{1}\right)=s^{\beta}$ and [7]

$$
Z^{*}\left[a, b_{1}\right]=p^{(2 p-1)} \Gamma(1-p) / \Gamma(p) .
$$

Thus, for the bound on $Z^{*}[a, b]=Z^{*}(\gamma, \mu, \nu)$ given in Theorem 4 and the error estimate for our approximation (36) given in Theorem 5 , we have[6]

$$
\eta_{X}\left(s ; a, b_{1}\right)=p^{(1-2 p)} T_{q}(S),
$$

where $S=2 p s^{1 / 2 p\rangle}, q=1-p$, and $T_{q}$ denotes a Lanchester-Clifford-Schläfli (LCS) function, which is analogous to the hyperbolic tangent (see Table 1). These functions were introduced in [12] and redefined for reasons of force-annihilation prediction in [6]. In fact,

$$
\eta\left(s ; a, b_{1}\right)=\tanh s, \text { when } \mu=\nu .
$$

This result is one of our reasons for introducing the normal form (7).

We have thus shown that the following theorem holds.

Table 1. Lanchester-Clifford-Schläfli functions

$$
\begin{aligned}
& F_{\alpha}(x)=\Gamma(\alpha) \sum_{k=0}^{\infty} \frac{(x / 2)^{2 k}}{k ! \Gamma(k+\alpha)} \\
& H_{\alpha}(x)=\Gamma(\alpha) \sum_{k=0}^{\infty} \frac{(x / 2)^{2(k+\alpha)}}{k ! \Gamma(k+\alpha+1)} \\
& T_{\alpha}(x)=H_{1-\alpha}(x) / F_{\alpha}(x)
\end{aligned}
$$

Relation to normal-form GLF

$$
\begin{array}{ll}
c_{X}(s)=F_{q}(S) & s_{X}(s)=p^{(1-2 p)} H_{p}(S) \\
c_{Y}(s)=F_{p}(S) & s_{Y}(s)=p^{(2 p-1)} H_{q}(S) \\
\hline \text { where } q=1-p \text { and } &
\end{array}
$$

$$
S(s)=2 p s^{1 /(2 p)}
$$

NOTE: For $\mu=\nu$, we have

(I) $c_{X}(s)=c_{Y}(s)=F_{1 / 2}(s)=\cosh s$,

(II) $s_{X}(s)=s_{Y}(s)=H_{1 / 2}(s)=\sinh s$,

(III) $\eta_{X}(s)=\eta_{Y}(s)=T_{1 / 2}(s)=$ tanh $s$. 


\section{Theorem 6}

Assume that either $\mu>-1$ or $\nu>-1$. Then for a battle modelled with the offset power attrition-rate coefficients (39), bounds on the modified parity-condition parameter $Z^{*}(\gamma, \mu, \nu)$ are given for $\gamma>0$ by

$$
\eta_{X}(s ; \gamma, \mu, \nu)<\frac{1}{Z^{*}(\gamma, \mu, \nu)}<\eta_{X}(s ; \gamma, \mu, \nu)+p^{q-p}\left\{\frac{\Gamma(p)}{\Gamma(q)}-T_{q}(S)\right\},
$$

where $q=1-p, S=2 p s^{1 /(2 p)}$, and $\eta_{X}(s ; \gamma, \mu, \nu)$ denotes the quotient of two normal-form hyperbolic-like GLF for the attrition-rate coefficients (39), i.e. $\eta_{X}(s ; \gamma, \mu, \nu)=s_{X}(s ; \gamma, \mu, \nu)$ ! $c_{X}(s ; \gamma, \mu, \nu)$.

It follows from Theorem 6 (or, equivalently, Theorem 4) that if we approximate $Z^{*}(\gamma, \mu, \nu)$ with $\hat{Z}(\hat{s} ; \gamma, \mu, \nu)$ defined by

$$
\hat{Z}(\hat{s} ; \gamma, \mu, \nu)=1 / \eta_{X}(\hat{s} ; \gamma, \mu, \nu),
$$

then bounds on the fractional error made in this estimate are given by

$$
0<f_{E}(\hat{s})<p^{q-p}\left\{\frac{\Gamma(p)}{\Gamma(q)}-T_{q}(S)\right\} \eta_{X}(\hat{s} ; \gamma, \mu, \nu)
$$

where $f_{E}(\hat{s})$ denotes the fractional error and is defined by (37).

The right-hand inequality in (49) [equivalently, (47)] tells us exactly how large to take $\hat{s}$ for the estimation of $Z^{*}(\gamma>0, \mu, \nu)$ by $Z(\hat{s} ; \gamma, \mu, \nu)$ to any specified degree of accuracy. The LCS function $T_{q}$ is involved in the bound on the fractional error $f_{E}(\hat{s})$ in this estimate when $\mu \neq \nu$. As (46) and Table 1 show, $T_{q}(S)=\tanh s$ when $\mu=\nu$. Thus, the LCS functions as redefined by Taylor and Brown [6] yield valuable information about battles modelled with not only the power attrition-rate coefficients with no offset (43) but also the offset power attrition-rate coefficients (39). Availability of tabulations of these LCS functions is discussed in [6].

\section{NUMERICAL RESULTS}

In this section we will examine several numerical examples to show how the modified parity-condition parameter $Z^{*}$ may be numerically determined and to show some important insights into the dynamics of combat that may be consequently obtained. In order to numerically determine the modified parity-condition parameter for the offset power attrition-rate coefficients (39), we must use knowledge about how quickly the limiting value (i.e. $Z^{*}\left[a, b_{1}\right]$ ) of a hyperbolic-tangent-like function of a related pair of power attrition-rate coefficients with "no offset" (43) is reached as its argument is increased [recall Theorem 6 and (49)]. In Fig. 3 we see

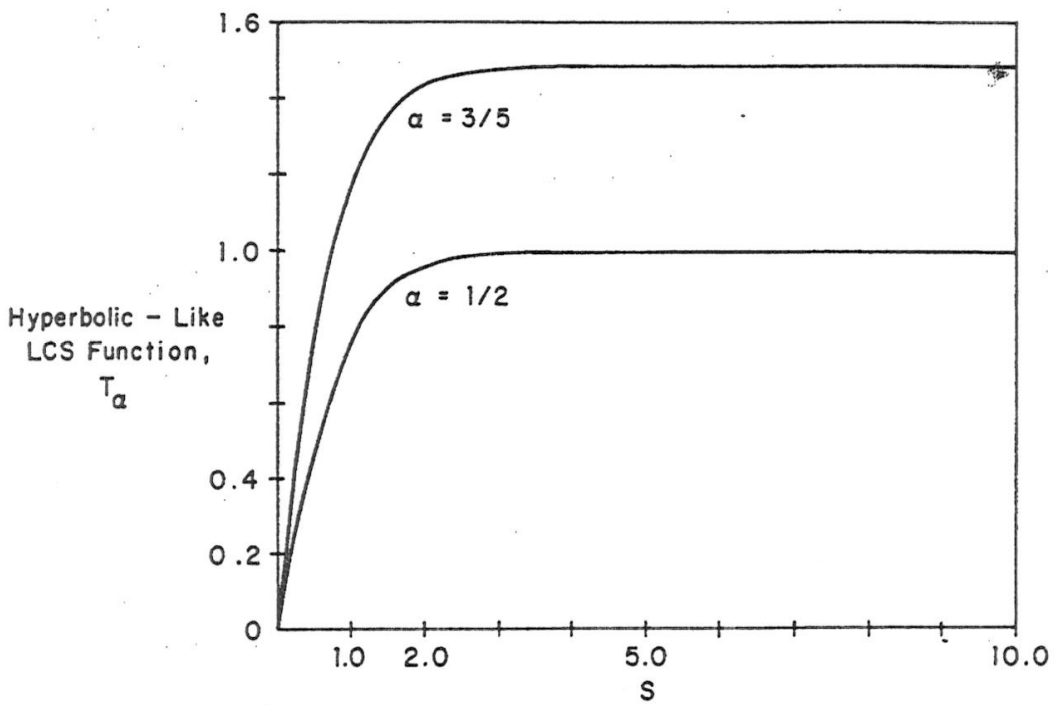

Fig. 3. Rapidity with which limiting value of hyperbolic-tangent-like LCS function $T_{\alpha}(S)$ is reached as $S \rightarrow+\infty$. Note: $T_{\alpha}(S)=\tanh s$ for $\alpha=1 / 2$, which corresponds to $\mu=\nu$ in (43). 


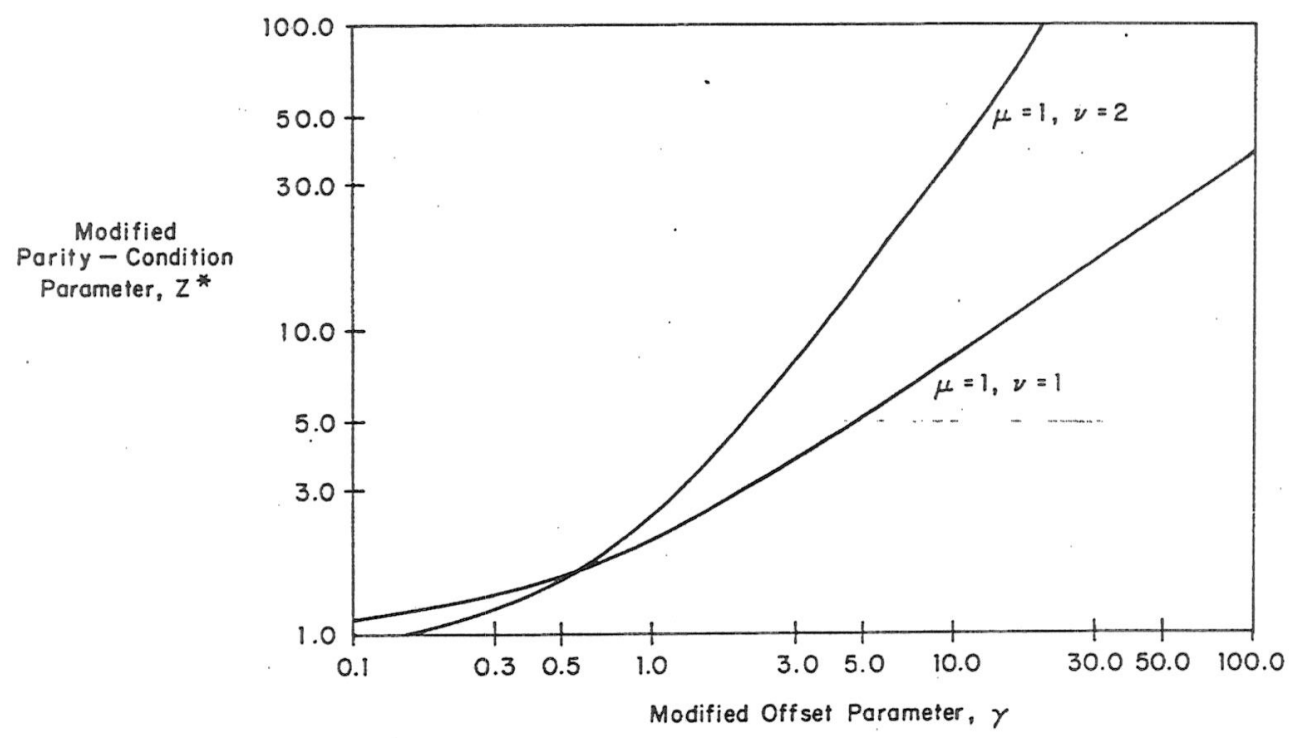

Fig. 4. Dependence of the modified parity-condition parameter $Z^{*}$ on the modified offset parameter $\gamma$ for the offset power attrition-rate coefficients. The modified offset parameter is given by $\gamma=A \cdot\left[\lambda_{I} /(\mu+1)\right]^{2 / 2}$, where $A$ is the off set parameter and $\Sigma=\mu+\nu+2$.

that this limiting value, denoted as $Z^{*}(\mu, \nu)=Z^{*}\left[a, b_{1}\right]$, is quite quickly reached: if one takes $\hat{s}=10.0$, then $Z^{*}(\mu, \nu)$ is approximated to better than six decimal places by $\hat{Z}(\hat{s} ; \mu, \nu)=$ $1 / \eta_{X}(\hat{s} ; \mu, \nu)$, where $\eta_{X}$ is given by (45). Experimental computing for various values of $\mu$ and $\nu$ and comparison with the known value (44) for $Z^{*}(\mu, \nu)$ bears out this degree of accuracy [i.e. speed of convergence of $\hat{Z}(\hat{s} ; \mu, \nu)$ to $Z^{*}$ ] for essentially all allowable values of $\mu$ and $\nu$. Thus, $\hat{Z}(\hat{s} ; \mu, \nu)$ for the coefficients (43) has essentially converged to $Z^{*}(\mu, \nu)$ when $\hat{s}=10.0$, and by Theorem 6 or (49) we know that the same is true for $\hat{Z}(\hat{s} ; \gamma, \mu, \nu)$ for the coefficients (39).

We have accordingly generated by this procedure the results shown in Fig. 4. For computing $\eta_{X}=s_{X} / c_{X}$, we have used the series solutions shown in Tables 2 and 3. (In Tables 2 and 3 we have for convenience denoted, for example, $s_{X}(s ; \gamma, \mu, \nu)$ simply as $s_{X}(s ; \mu, \nu)$, i.e. $s_{X}(s ; \mu, \nu)$ denotes $s_{X}$ corresponding to the general power attrition-rate coefficients (9) with exponents $\mu$ and $\nu$.) The series were obtained by solving (17) by the method of successive approximations (see [23]). We used these series instead of developing approximate solutions by finite-difference methods because we did not have any error bounds for the latter.

Let us now give an intuitive interpretation of the curves shown in Fig. 4 of the modified parity-condition parameter $Z^{*}$ plotted vs the modified offset parameter $\gamma$. In Taylor and Comstock [7] it is shown that $Z^{*}$ may be considered to be the initial $Y$ force level that leads to a

Table 2. Normal-form offset linear Lanchester functions

$$
\begin{aligned}
& c_{X}(s ; 1,1)=\sum_{k=0}^{\infty} \frac{s^{2 k}}{(2 k) !} \sum_{j=0}^{k} A_{k}^{j}\left(\frac{\gamma}{\sqrt{ }(s)}\right)^{j} \\
& s_{X}(s ; 1,1)=\sum_{k=0}^{\infty} \frac{s^{2 k+1}}{(2 k+1) !} \sum_{j=0}^{k} B_{k}^{i}\left(\frac{\gamma}{\sqrt{ }(s)}\right)^{j}
\end{aligned}
$$

where the offset coefficients are given by*

$$
\begin{aligned}
& \left\{\begin{array}{l}
A_{0}{ }^{0}=1, \text { and for } k \geq 1 \\
A_{k}{ }^{j}=\frac{4 k(4 k-2)}{(4 k-j)(4 k-2-j)}\left\{A_{k-1}^{j}+A_{k-1}^{j-1}\right\}
\end{array} \text { for } 0 \leq j \leq k\right. \\
& \left\{\begin{array}{l}
B_{0}{ }^{0}=1, \quad \text { and for } \quad k \geq 1 \\
B_{k}{ }^{j}=\frac{4 k(4 k+2)}{(4 k-j)(4 k+2-j)}\left\{B_{k-1}^{j}+B_{k-1}^{j-1}\right\}
\end{array} \text { for } 0 \leq j \leq k\right.
\end{aligned}
$$

*We have adopted the convention that $A_{k}^{j}, B_{k}^{j}=0$ for $j<0$ or $j>k$. 
Table 3. Offset power Lanchester functions for $\mu=1$ and $\nu=2$

$$
\begin{aligned}
& \left\{\begin{array}{l}
A_{0}{ }^{0}=1, \quad \text { and for } \quad k \geq 1 \\
A_{k}^{j}=\frac{5 k(5 k-2)}{(5 k-j)(5 k-2-j)}\left\{A_{k-1}^{j-2}+2 A_{k-1}^{j-1}+A_{k-1}^{j}\right\} \text { for } 0 \leq j \leq 2 k
\end{array}\right. \\
& \left\{\begin{array}{l}
B_{0}^{0}=1, \quad \text { and for } \quad k \geq 1 \\
B_{k}^{j}=\frac{5 k(5 k+2)}{(5 k-j)(5 k+2-j)}\left\{B_{k-1}^{j-2}+2 B_{k-1}^{j-1}+B_{k-1}\right\} \quad \text { for } \quad 0 \leq j \leq 2 k
\end{array}\right.
\end{aligned}
$$

*We have adopted the convention that $A_{k}^{i}, B_{k}{ }^{j}=0$ for $j<0$ or $j>2 k$.

draw* (i.e. parity between the forces) in the battle against an $X$ force of "unit strength"

$$
\begin{array}{ll}
\mathrm{d} x / \mathrm{d} s=-y & \text { with } x(s=0)=1, \\
\mathrm{~d} y / \mathrm{d} s=-J(s) x & \text { with } y(s=0)=Z^{*},
\end{array}
$$

where $J(s)$ denotes the invariant of the normal form (17). Thus, we may consider $Z^{*}$ to be "the $Y$ equivalent of an $X$ force of unit strength" for the modified battle (50). Now let us consider the general power attrition-rate coefficients (9) with exponents $\mu$ and $\nu$. As we did in Tables 2 and 3 , we will denote the corresponding $J$ as $J(s ; \mu, \nu)$ and $Z^{*}(\mu, \nu)$ to stress the dependence on $\mu$ and $\nu$ (but suppressing that on $\gamma$ ). We then have from (42) that $J(s ; 1,1)=1+\gamma / \sqrt{ }(s)$ and $J(s ; 1,2)=\sqrt{ }(s)(1+\gamma / \sqrt{ }(s))^{2}$. From (44) we find that $Z^{*}(1,1)=1.000$ and $Z^{*}(1,2)=0.806$ for $\gamma=0$. Observing that for $\gamma>1$ we have $J(s ; 1,1)<J(s ; 1,2)$ for all $s \geq 0$, it is intuitatively clear from (50) and the interpretation of $Z^{*}$ as a force equivalent that we must have $Z^{*}(1,1)<$ $Z^{*}(1,2)$ for all $\gamma>1$ because $X$ always has greater fire effectiveness against $Y$.when $\mu=1$ and $\nu=2$ than when $\mu=1$ and $\nu=1$. However, for $\gamma$ near zero, the situation is reversed and $Z^{*}(1,2)$ must lie below $Z^{*}(1,1)$ for $\gamma$ near zero. Thus, we have given an intuitive explanation of why $Z^{*}(1,2)$ lies below $Z^{*}(1,1)$ for $\gamma$ near zero but above it for $\gamma>1$ as Fig. 4 shows.

Next, we will consider numerical results for a particular battle to show some of the important insights that may be gained into the dynamics of combat from our new.results. As in $[6,12,23]$ we consider S. Bonder's $[3,19]$ model $(10)$ for the constant-speed attack of mobile forces against a static defensive position. We will focus on the new results given in this paper (in particuular, the prediction of battle outcome from initial conditions without explicitly computing the force-level trajectories). Input data and computed parameter values are shown in Table 4. We will now consider two cases: (I) $R_{0}=1500 \mathrm{~m}$; and (II) $R_{0}=1250 \mathrm{~m}$.

When $R_{0}=1500 \mathrm{~m}$, we have $C=0$ and $s_{0}=0$. The maximum time that the battle can last is $t_{\max }=11.18 \mathrm{~min}$, since at this time the advancing attackers overrun the defensive position. In this case $Z^{*}(\gamma, \mu, \nu)=Z^{*}(0.32,1.1)=1.381$, so that Theorem 2 tells us that $X$ can be annihilated $<==>x_{0} / y_{0}<0.264$. By (30) the $X$-force annihilation time is given by $\eta_{X}\left(s\left(t_{a}{ }^{X}\right)\right)=2.739 x_{0} / y_{0}$. For $x_{0}=10$ and $y_{0}=50$, we have $\eta_{X}\left(s_{a}{ }^{X}\right)=0.54772$ so that by the techniques introduced in [6] we find $s_{a}{ }^{X}=0.771$. These computations for determining $s_{a}{ }^{X}$ involve the generation of a table of $s_{X}, c_{X}$, and $\eta_{X}$ for $\gamma=0.32, \mu=\nu=1$ (see [6]). Hence, (36) yields $t_{a}{ }^{X}=10.25 \mathrm{~min}$ and $r_{a}^{X}=125.7 \mathrm{~m}$. Further results are given in Table 5 .

When $R_{0}=1250 \mathrm{~m}$ (see Fig. 5 of [12]), we have $C=1.864 \mathrm{~min}, s_{0}=0.0255$ and $t_{\max }=9.32 \mathrm{~min}$.

*In other words, $x(s)$ and $y(s)>0$ for all $s \in[0,+\infty)$ but $\lim _{s \rightarrow+\infty} x(s)=0=\lim _{s \rightarrow+\infty} y(s)$. 
Table 4. Particulars for the numerical examples

1. Input data

$\mu=\nu=1$

$\alpha_{0}=0.06 X$ casualties $/ \mathrm{min} / Y$ unit

$\beta_{0}=0.6 Y$ casualties $/ \mathrm{min} / X$ unit

$R_{\alpha}=1500 \mathrm{~m}, R_{\beta}=2000 \mathrm{~m}$

$v=5$ miles $/ \mathrm{hr}$

2. Parameter values

$k_{a}=5.364 \times 10^{-3} X$ casualties $/ \mathrm{min} / Y$ unit

$k_{b}=4.023 \times 10^{-3} Y$ casualties $/ \mathrm{min} / X$ unit

$p=q=1 / 2$

$A=3.728 \mathrm{~min}, \gamma=0.320$ (casualties $/ \mathrm{min})^{1 / 2}$
Table 5. Annihilation of the $X$ force as a function of the initial force ratio for $R_{0}=1500 \mathrm{~m}$

\begin{tabular}{lcc}
\hline$\left(x_{0} / y_{0}\right)$ & $t_{a}{ }^{x}(\min )$ & $r_{a}{ }^{X}(\mathrm{~m})$ \\
\hline 0.250 & 14.09 & $\overline{-}^{*}$ \\
0.200 & 10.25 & 125.7 \\
0.167 & 8.80 & 319.4 \\
\hline
\end{tabular}

${ }^{*} t_{\max }=11.18 \min$ and $x_{f}=x(r=0)=2.48$.

Table 6. Annihilation of the $X$ force as a function of the initial force ratio for $R_{0}=1250 \mathrm{~m}$

\begin{tabular}{ccc}
\hline$\left(x_{0} / y_{0}\right)$ & $t_{a}{ }^{X}(\min )$ & $r_{a}{ }^{x}(\mathrm{~m})$ \\
\hline 0.250 & 10.87 & $-\overline{-}^{*}$ \\
0.200 & 8.17 & 154.4 \\
0.167 & 6.93 & 320.4 \\
\hline
\end{tabular}

${ }^{*} t_{\max }=9.32 \min$ and $x_{f}=x(r=0)=1.74$.

In this case $X$ can be annihilated $<==>x_{0} / y_{0}<0.281$ with the $X$-force annihilation time given by $\eta_{X}\left(s_{a}{ }^{X}\right)=\left(1.001 u_{0}+0.009\right) /\left(0.127 u_{0}+0.366\right)$, where $u_{0}=x_{0} / y_{0}$. Numerical results are given in Table 6 . Finally, these parametric results should be contrasted to those previously possible (e.g. compare them with, for example, the single force-level trajectory for $R_{\beta}=2000 \mathrm{~m}$ shown in Fig. 5 of [12].

\section{DISCUSSION}

S. Bonder $[3,18,19]$ has emphasized the shortcomings of constant-coefficient Lanchestertype combat models. Work by Bonder $[3,18]$, Clark[25], and others [4] on the prediction of Lanchester attrition-rate coefficients (see Taylor and Brown[12] for further discussion and references) has generated interest in variable-coefficient models. Moreover, there is not only intrinsic interest (see $[3,19]$ ) in the model $(7)$ but also interest for obtaining insights into the behavior of complex Lanchester-type system models (for example, the Bonder-IUA model (see $[4,5,14])$ ) that have been enriched in military detail see $[4,5,14-17])$. The attrition-rate coefficients in (7) represent the fire effectiveness of the combatants and allow us to model temporal variations in fire effectiveness on the battlefield. Interest in the general power attrition-rate coefficients (9) is provided by S. Bonder's $[3,5,19]$ constarlt-speed attack model* (10), (11) and his examination of the range dependence of attrition-rate coefficients for various weapon systems (see pp. 196-200 of [4]).

We have given results that allow one to study the variable-coefficient model (7) (especially with the general power attrition-rate coefficients (9)) almost as easily and thoroughly as Lanchester's classic constant-coefficient model (1). Taylor and Comstock [7] (see Theorems 1 and 2 above) have shown how to predict force annihilation without having to spend the time and effort of explicitly computing force-level trajectories. Using their theoretical results, we gave results in a previous paper [6] that made combat modelled by power attrition-rate coefficients with no offset $\dagger$ [i.e. $A=0$ in (9)] almost as easy to analyze as the constantcoefficient case. The results of the paper at hand allow one to analyze combat modelled by power attrition-rate coefficients with positive offset‡ [i.e. $A>0$ in (9)] just as conveniently.

Theorem 1 (see also Theorem 2) is the generalization of the classic constant-coefficient result (5) to cases of time-dependent attrition-rate coefficients. However, one needs to know the

*Thus, the range between firer and target changes during the engagement.

†Modelling, for example, combat between two weapon systems with the same maximum effective range.

$\ddagger$ Modelling, for example, combat between two weapon systems with different maximum effective ranges. 
value of the so-called parity-condition parameter $Q^{*}$ in order to predict force annihilation in specific instances. In this paper we have presented theoretical considerations (see Section 5 above) for the noniterative numerical determination of the parity-condition parameter. We applied our general theory to the specific case of general power attrition-rate coefficients (9) (see Section 6) and illustrated these theoretical results by considering some numerical examples (see Section 7).

Curves of the modified parity-condition parameter $Z^{*}$ plotted against the modified offset parameter $\gamma$ such as those shown in Fig. 4 allow one to analyze parametrically "modern" combat modelled with the general power attrition-rate coefficients (9). For example, we can now parametrically (for example, varying the maximum effective range of the defender's.weapons) determine whether the defender will be overrun in Bonder's $[3,5,19]$ constant-speed-attack model (10) with attrition-rate coefficients (11) without having to compute the entire force-level trajectories. We have illustrated this analysis capability with some numerical examples, which show that the defender's annihilation (i.e. saturation of his defensive position with offensive fire) depends on the initial force ratio (of defender to attacker) being below a certain threshold value. Our new results allow one not only to determine easily such force-ratio thresholds of survivability but also to study their dependence on weapon-system-capability parameters.

Our new results let us conveniently obtain much valuable information about the model (7). The classic ordinary differential equation theories (see, for example, Ince[26] were inadequate to answer many important questions (for example, "who will win? Be annihilated?") about such combat models. Previously one was limited to only computing force-level trajectories, but now we can predict battle outcome (in particular, force annihilation) without explicitly computing force-level trajectories. Moreover, these new results facilitate parametric analysis of such combat situations. S. Bonder [22] has suggested that an increased emphasis be place on parametric analyses in systems analysis studies (see pp. 21-22 of [22]). In particular, Theorems 1 and 2 explicitly exhibit a tradeoff between quality (as quantified by the relative-fireeffectiveness parameter $\lambda_{R}$ and the parity-condition parameter $Q^{*}$ ) and quantity (as quantified by the initial force ratio $x_{0} / y_{0}$ ) of two weapon systems in combat against each other. In other words, one can use an expression like (21) to develop quantitative insights into how the quality of a weapon system may be substituted for sheer numbers. Moreover, an unanswered theoretical question is to determine how the parity-condition parameter $Q^{*}$ depends on the combat-intensity parameter $\lambda_{I}$ and the relative-fire-effectiveness parameter $\lambda_{R}$. Finally, our results here are signposts as to the difficulty of analytically extracting information (particularly parametric information without excessive computations) from variable-coefficient Lanchestertype models such as (7).

\section{REFERENCES}

1. L. Dolansky, Present state of the Lanchester theory of combat, Ops Res., 12, 344-358 (1964).

2. F. W. Lanchester, Aircraft in warfare: the dawn of the fourth arm-No. V., the principle of concentration, Engineering, 98, 422-423 (1914).

3. S. Bonder, A Theory for Weapon System Analysis, Proc. U.S. Army Ops. Res. Symp., 4, 111-128 (1965).

4. S. Bonder and R. Farrell, (Eds.), Development of Models for Defense Systems Planning, Report No. SRL 2147 TR $70-2$ (U), Systems Research Laboratory, The University of Michigan, Ann Arbor, Michigan (1970).

5. S. Bonder and J. Honig, An Analytical Model of Ground Combat: Design and Application, Proc. U.S. Army Ops. Res. Symp., 10, 319-394 (1971).

6. J. G. Taylor and G. G. Brown, Further Canonical Methods in the Solution of Variable-Coefficient Lanchester-type Equations of Modern Warfare: a New Definition of Power Lanchester Functions, Tech. Report No. NPS55-27, Naval Postgraduate School, Monterey, California (1977) (AD A044 302); also submitted to Ops. Res.

7. J. G. Taylor and C. Comstock, Force-annihilation Conditions for Variable-coefficient Lanchester-type Equations of Modern Warfare, Nav. Res. Logist. Q., 24, 349-371 (1977).

8. J. G. Taylor and S. H. Parry, Force-ratio Considerations for Some Lanchester-type Models of Warfare, Ops. Res., 23, 522-533 (1975).

9. H. K. Weiss, Requirements for a Theory of Combat, Memorandum Report No. 667, Ballistic Research Laboratories, Aberdeen Proving Ground, Maryland (1953).

10. H. K. Weiss, Lanchester-type Models of Warfare, in Proc. First Int. Conf. on Operational Research, (Edited by M. Davies, R. T. Eddison, and T. Page), pp. 82-98. Operations Research Society of America, Baltimore (1957).

11. H. K. Weiss, Some Differential Games of Tactical Interest and the Value of a Supporting Weapon System, Ops. Res., 7, 180-196 (1959).

12. J. G. Taylor and G. G. Brown, Canonical Methods in the Solution of Variable-Coefficient Lanchester-type Equations of Modern Warfare, Ops. Res., 24, 44-69 (1976).

13. S. Bonder, An Overview of Land Battle Modeling in the U.S., Proc. U.S. Army Ops. Res. Symp., 13, 73-88 (1974). 
14. S. Bostwick, F. Brandi, C. Burnham and J. Hurt, The Interface between DYNTACS-X and Bonder-IUA, Proc. U.S. Army Ops. Res. Symp., 13, 494-502 (1974).

15. W. P. Cherry, The Role of Differential Models of Combat in Fire Support Analysis, in Fire Support Requirements Methodology Study Phase II, Proceedings of the Fire Support Methodology Workshop, Appendix 4, Ketron, Arlington, Virginia (1975).

16. A. Cordesman, (Ed.), Developments in Theater Level War Games, unpublished materials for C-5 Working Group of 35th Military Operations Research Symposium (1975).

17. R. Farrell, Vector 1 and Battle: Two Versions of a High-resolution Ground and Air Theater Campaign Model, in Military Strategy and Tactics (Edited by R. Huber, L. Jones and E. Reine), pp. 233-241, Plenum Press, New York (1975).

18. S. Bonder, The Lanchester Attrition-rate Coefficient, Ops Res., 15, 221-232 (1967).

19. S. Bonder, A Model of Dynamic Combat, in Topics in Military Operations Research, pp. IV-I to IV-37, The University of Michigan Engineering Summer Conferences, 21 July-1 August 1969.

20. J. G. Taylor, Target Selection in Lanchester Combat: Heterogeneous Forces and Time-dependent Attrition-rate Coefficients, Nav. Res. Logist. Q., 21, 683-704 (1974).

21. J. G. Taylor, On the Treatment of Force-Level Constraints in Time-sequential Combat Problems, Nav. Res. Logist. Q., 22, 617-650 (1975).

22. S. Bonder, Systems Analysis: a Purely Intellectual Activity, Milit. Rev., 51(2), 14-23 (1971).

23. J. G. Taylor, Solving Lanchester-type Equations for "Modern Warfare" with Variable Coefficients, Ops. Res., 22, 756-770 (1974).

24. S. Bonder, The Mean Lanchester Attrition Rate, Ops. Res., 18, 179-181 (1970).

25. G. M. Clark, The Combat Analysis Model, Ph.D. thesis, The Ohio State University, Columbus, Ohio (1969).

26. E. L. Ince, Ordinary Differential Equations, Longmans-Green, London (1927); reprinted by Dover, New York (1956). 